\title{
TEORÍA CONTABLE: FUNDAMENTO DE ANÁLISIS EN EL EJERCICIO PROFESIONAL Y LA
}

\author{
PRÁCTICA PEDAGÓGICA*
}

ACCOUNTING THEORY: ANALYSIS TOOLIN PROFESSIONAL PRACTICE AND TEACHING PRACTICE

\author{
Yuleida Ariza Angarita** \\ Milagros Villasmil Molero ${ }^{* * *}$
}

RECIBIDO: Marzo 5 de 2014

ACEPTADO: Mayo 20 de 2014

\section{RESUMEN}

El presente artículo de revisión bibliográfica, se realizó con el objetivo de analizar la teoría contable con fundamento en el análisis del ejercicio profesional y la práctica pedagógica en la formación integral del contador público. En su elaboración, se utilizó el método analítico, con una visión descriptiva, y la técnica investigativa de la revisión documental. Entre los hallazgos obtenidos, se destaca que en el proceso formativo del contador aún se encuentran poco desarrolladas las competencias de observación, reflexión analítica y predicción, por cuanto, al momento de tomar decisiones se le da más importancia a la parte cuantitativa de la información que a la parte cualitativa . Las razones obedecen en gran parte a débiles bases conceptuales acerca de su valor y su contribución al desarrollo de nuevas prácticas y pensamiento contable. Se concluyó que para que a la teoría contable le sea reconocido su valor proyectivo y predictivo, es imperativo una pedagogía particular para estudiarla, comprenderla e introducirla como fundamento de soporte esencial para el análisis financiero en el ejercicio de la profesión contable; ello es así porque las cambiantes visiones de la realidad exigen nuevas formas de observación fenoménica, que favorezcan la solución a problemas complejos que le son inherentes. Asimismo, se espera que el estudio bibliográfico constituya un asidero epistemológico para futuras investigaciones.

Palabras clave: Teoría contable, contador público, ejercicio profesional, práctica pedagógica.

Este artículo se debe referenciar así:

Ariza, Y. \& Villasmil M. (2014). Teoría contable: fundamento de análisis en el ejercicio profesional y la práctica pedagógica, en Desarrollo Gerencial, Revista de la Facultad de Ciencias Económicas, Administrativas y Contables de la Universidad Simón Bolívar. 6 (2). pp $41-71$.

\footnotetext{
*Este artículo es producto de los resultados parciales del proyecto de investigación: El estudio de la teoría contable: Mitos y realidades en el proceso de enseñanza-aprendizaje de la disciplina en universidades de gestión privada desde la visión colombo-venezolana, financiado por el Centro de Investigación y Extensión de Ciencias Económicas y Contables (CIECEC) de la Universidad de la Costa, CUC.

**Contador Público, Especialista en Sistemas de Gestión de Calidad Integrados, Magister en Educación, Docente Investigador Universidad la Costa CUC, Universidad Simón Bolívar. Correo electrónico: yuliari74@hotmail.com ***Contador Público, Posdoctoral en Gerencia Publica y Gobierno, doctora en Ciencias Gerenciales, Magister en Gerencia Tributaria, docente investigador de la Universidad de a Costa CUC. Correo electrónico: milagrosv28@gmail.com.
} 


\begin{abstract}
This article literature review was conducted with the aim of analyzing accounting theory as a foundation in the analysis of professional practice and pedagogical practice in the formation of the CPA, in drafting the analytical method was used, with a descriptive view technical research is the literature review. Among the findings from the formative process of the counter are still lagging skills of observation, analysis and reflection-prediction, in that he gives more importance to the quantitative portion of the information that highlights the qualitative part for decision making, the reasons are due largely to weak conceptual basis about its value against the correct usage you have and its contribution to the development of new practices and accounting thought. It was concluded that for accounting theory is recognized its projective and predictive value, it is imperative to study a particular pedagogy understand and enter it as essential foundation for financial analysis support in the performance of the accounting profession; considering that this paradigmatic changing views of reality require new forms of phenomenal observation that favors the solution to complex problems that are inherent, also is expected to constitute an epistemological journey bibliographic handle foundation for future research.
\end{abstract}

Key words: Accounting theory, public accountant, professional practice, teaching practice. 


\section{INTRODUCCIÓN}

El estudio de la teoría contable parece una tarea tediosa, intrascendente y poco operativa; sin embargo, conociendo las bases, prácticas primarias y la evolución de la contabilidad en el transcurrir de los tiempos, se puede dar sentido y entender las doctrinas contables del presente. Por ello, rebuscar sus fuentes en el pasado, para conocer la razón de los procedimientos que son aplicados hoy, es proyectar el futuro de la profesión, lo cual no es solo un deber de todo contador, sino un imperativo para el avance de la ciencia. Sobre todo, cuando es evidente la débil trascendencia de los conocimientos que genera la investigación en esta área.

Justamente, este documento pretende mostrar las débiles relaciones de la teoría contable con el ejercicio profesional del contador público y su poca correspondencia con el proceso formativo del mismo. Todo esto, con el ánimo de destacar el valor de este saber en la formación integral del profesional contable, dada la importancia que debiera tener en la toma de decisiones del área.

Explorar los problemas que enfrenta la contabilidad como disciplina social permite discernir, entonces, su compleja realidad humana e identificar formas de pensar y presupuestos teóricos que despierten interés en nuevas investigaciones y metodologías para su enseñabilidad, eje central de la presente investigación.

Ya entrando en materia, si se considera que el concepto de teoría contable tiene diversos significados, y que esta disciplina ha sido demarcada por fuertes influencias ideológicas, políticas y filosóficas desde sus inicios, el presente estudio posibilita reflexionar en torno a 
las siguientes preguntas: ¿Cuál es la Importancia de la teoría contable en los procesos formativos del contador público? y ¿cuáles son las implicaciones de la teoría contable en el ejercicio profesional y la práctica pedagógica de cara a los desafíos de la sociedad actual?

\section{MÉTODO}

El presente artículo se inserta en el campo de los problemas educativos referidos a la enseñabilidad de la contabilidad, campo en el que el método inductivo permite analizar e interpretar el fenómeno en contexto, toda vez que la teoría contable constituye una reflexión en y desde la praxis, y su práctica pedagógica está constituida por hechos observables externos y por símbolos e interpretaciones elaborados a partir del medio en que interviene.

\section{Diseño}

El estudio es de tipo documental analítico, y se realizó desde la visión exploratoria descriptiva, con diseño de corte bibliográfico, bajo la mirada crítica de la acción educativa y buscando posibilidades de intervención. En concreto, se pretende mirar la profesión contable desde un enfoque formativo para reflexionar sobre sus prácticas pedagógicas e intentar mejorarlas, de modo que los profesionales del área respondan adecuadamente a las exigencias del contexto local y de la sociedad global.

\section{Instrumentos}

La presente investigación empleó como técnica para el análisis cualitativo de las categorías: el ejercicio profesional y práctica pedagógica, la elaboración de fichas de trabajo, tomando en cuenta la fuente, su autor, año y contenido relevante para el tema. Fue así como se seleccionó la información pertinente al 
objeto de estudio, permitiendo el logro de los objetivos planteados.

\section{Procedimientos}

Para llevar a cabo el presente estudio, se realizó un proceso de selección y delimitación del tema de investigación y de los documentos relacionados, lo cual se circunscribió al análisis de las categorías: ejercicio profesional y la práctica pedagógica en el contexto de la enseñanza-aprendizaje de la Teoría Contable. Este análisis se apoyó en fuentes primarias $\mathrm{y}$ secundarias, a través de la investigación documental en bibliotecas, hemerotecas, archivos, páginas electrónicas, entre otros. Se revisaron doctrinas, textos, revistas y publicaciones en materia de la teoría contable y, específicamente, en el proceso de enseñabilidad de la disciplina desde la práctica pedagógica y el ejercicio profesional.

\section{RESULTADOS}

Los resultados atienden los criterios teóricos considerados en la teoría contable y comportan un método analítico interpretativo. En ellos se demuestra que la teoría contable representa un soporte estratégico de análisis en el ejercicio profesional y la práctica pedagógica. Es de resaltar que la revisión, tanto de currículos como de las prácticas educativas, constituye una necesidad permanente de los programas de formación del contador, debido a la vorágine de cambios que experimenta la sociedad globalizada, la cual reclama un profesional flexiblemente adaptado y con visión universal a fin de que entienda lo social desde lo humano (Morín, 1999).

Enseguida se presentan los resultados, de acuerdo con las categorías consideradas en el presente trabajo investigativo: 
La teoría contable, desde el ejercicio profesional, requiere que las intervenciones en los hechos económicos se hagan con criterios abiertos y reflexivos, que, soportados por análisis retrospectivos y proyectivos, favorezcan la toma de decisiones sustentadas en evaluaciones de impactos. Estas intervenciones también deben ofrecer múltiples opciones a problemas complejos y cambiantes, constituyéndose así en un fundamento útil, capaz de satisfacer las necesidades de gestión contable de las organizaciones y de la sociedad en general.

\section{La teoría contable, desde la} práctica pedagógica, representa una estrategia de análisis, en el proceso de enseñanza-aprendizaje, que genera nuevos enfoques de la realidad contable. En este sentido, en la actualidad se evidencia la necesidad de conminar a actores y estamentos del proceso educativo a la revisión y evaluación de sus concepciones de la teoría contable, así como las metodologías empleadas en su enseñanza. Ello implica un proceso alterno de formación docente que favorezca la importancia de su aplicación, de modo que su abordaje posibilite al profesional contable conocer desde adentro los fundamentos y axiomas de la contabilidad, para luego mirar hacia la práctica. Así sus actuaciones se proveerán de razones, dando explicación a hechos ocurridos y sirviendo de apoyo para la toma de decisiones, soportándose en otras disciplinas que amplíen su visión (Rojas, 1996).

Es claro que la teoría contable constituye el medio para nutrir $y$ promover el avance de la contabilidad como ciencia, por ello, las universidades $\mathrm{y}$ sus procesos formativos son los escenarios llamados a consolidar en sus profesionales una visión crítica reflexiva, 
con una actitud académica dispuesta a repensar sus teorías y métodos para perfeccionarlos, con el propósito de explicar y considerar de manera más holística los cambios de los contextos en los que interactúa. Todo esto involucra una nueva forma de investigar en escenarios multidimensionales y complejos, expuestos a constantes tensiones que requieren procesos de renovación y reconfiguración de dichos espacios para adecuarlos a las demandas actuales.

$$
\text { Por último, el recorrido } \mathrm{y}
$$

resultado del presente estudio conlleva una invitación para orientar nuevas investigaciones que impulsen el avance teórico de la contabilidad y entender a profundidad la concepción de esta disciplina. Por consiguiente, el nuevo paradigma contable exige ampliar la visión reduccionista de lo ya definido por la costumbre, para hacer nuevas construcciones cimentadas en conocimientos acordes con las realidades, en las que se apropie el verdadero uso de la teoría que, según Tua (1997), debe "descartar sistemas que no son contables, desarrollar otros que sí lo son y predecir el comportamiento de los sistemas contables" (p.342).

\section{DISCUSIÓN}

En las actualidad se percibe un desarraigo o ruptura entre la teoría contable y el ejercicio profesional del contador público, producto de un desconocimiento por parte de este de su verdadera aplicación; para superarlo, se debe partir de la comprensión de la disciplina contable como un todo, que integra teoría y práctica social, es decir, de una visión analítica y general guiada por la mirada de la complejidad, que favorece la integración y la relación. 
Cúrvelo (2009) indica al respecto

que:

La contabilidad debe servir primeramente para comprender y desarrollar una actitud crítica y reflexiva ante las prácticas económicas y administrativas, e incluso las contables, un poco lo que espera la sociedad y el Estado en términos de herramientas para el control social que promueve una participación en los procesos sociales que busca la justicia y el bien colectivo (p. 499).

En una dirección similar, Gracia (2002) plantea que la educación contable en Colombia se ha centrado en el campo de la instrumentación para formar habilidades y destrezas que demandan los medios productivos. Sin embargo, se presentan consideraciones que buscan dar a la contabilidad un sentido de ciencia social con injerencia directa en la dinámica de las organizaciones y los cambios del entorno (Tua, 1997). Ante esto, expresa Gómez (2012): “un contador activo en la transformación de la sociedad, requiere una enseñabilidad y educabilidad de la contabilidad cimentada en una teoría contable renovadora y emancipadora, delineada por el pensamiento crítico, puesto que dicha teoría es la orientadora de un ejercicio profesional en pos de la solución de los problemas sociales" (p. 99).

A tono con lo anterior, Morin (2011, p. 129) aduce que, de manera general, el modo en que se induce el conocimiento no permite desarrollar suficientemente la habilidad para contextualizar la información e integrarla de forma que tenga sentido y utilidad. "La enseñanza actual proporciona conocimientos sin enseñar qué es el conocimiento". Ello se debe a la fragmentación de saberes que no se relacionan e impiden concebir problemas complejos. En efecto, la teoría contable es concebida como el rudimento que describe la dinámica del ejercicio profesional limitándola al concepto escrito que detalla el registro y guía la 
práctica, pero ella también implica la interpretación de la realidad a partir de la comprensión de la teoría misma.

Schnitman (citado por Gómez, 2007) afirma que "la práctica es hacer el mundo a imagen y semejanza del modelo teórico", por lo que, justamente, al no usarse la teoría contable como fundamento de predicción sobre eventos ocurridos y no analizados, se pierde el propósito de servir de apoyo para la toma de decisiones, y no se logra el análisis crítico-reflexivo que requiere el correcto desarrollo de las prácticas y del pensamiento contable. De allí surge la contradicción planteada por Morin (2011) cuando afirma que las ciencias económicas separan el contexto político del universo físico y humano, y así pierden poder de predicción, a pesar de ser considerada la más exacta de las ciencias sociales.
De lo anterior se infiere que la enseñanza de la teoría debe partir de la conciencia de su importancia en la toma de decisiones a fin de "proveer razones para la práctica observada", además de "predecir", refiriéndose esto no solo a eventos futuros sino a fenómenos que han sucedido sin ser documentados. Sobre este particular, Gómez (2004), Cardona (2005) y Martínez (2002, p. 126) coinciden en que el sentido de la teoría contable se ha desviado hacia el enfoque jurídico pragmático, lo que favorece contenidos cargados de ejercicios prácticos y poca teoría contable "tendiendo a la pauperización del conocimiento". En estas circunstancias, el principal problema de la contabilidad es negar la necesidad de desarrollar el conocimiento teórico de la disciplina, quedándose en la simple reproducción del proceso contable (teneduría de libros). 
De igual forma, se observa que el enfoque pedagógico en la contabilidad se ha centrado en la operación, descuidándose la fundamentación teórica, que es necesaria para solucionarproblemas complejos. A raíz de este planteamiento, Lara (2002) menciona: "Uno no podría ser un buen profesional si no tiene un claro dominio de la disciplina, y de otro lado, tampoco sería un buen profesional aquel teórico que desconoce las prácticas de la misma" (p. 83).

Es oportuno retomar al respecto la la taxonomía de Bloom (1956), que hace referencia a las habilidades del pensamiento empleadas para jerarquizar los niveles del desarrollo humano y el dominio de determinado conocimiento.

Para lograr la apropiación significativa del mismo se requiere la aplicación de varias etapas, todas íntimamente ligadas, a saber: conocimiento básico, comprensión, aplicación, análisis, síntesis, emisión de juicios de valor y evaluación como evidencia de su funcionalidad. Han transcurrido más de 50 años y el método de Bloom continúa siendo un importante instrumento para establecer objetivos de aprendizaje.

De lo anterior, puede inferirse que la teoría contable se ha asumido como una simple memorización de conceptos, llegando apenas hasta el tercer nivel de la escala de Bloom (aplicación), lo cual hace que no promueva pensamiento crítico en los estudiantes. Ello sugiere que para que alcance el estatus del aprendizaje significativo deba estudiarse a través de razonamientos y aplicando criterios específicos, de forma que en verdad sustente el ejercicio profesional. Morín (2011) opina que: “deben enseñarse los métodos que permitan captar las relaciones mutuas, las influencias recíprocas, las inter-retro-acciones. Jamás 
accederemos a un conocimiento total: el todo del universo siempre será inaccesible. Pero debemos aspirar, por lo menos, a un conocimiento multidimensional" (p. 152).

Cabe señalar que la anhelada visión polifacética de la contabilidad es un tema complicado de analizar y discutir por el carácter cambiante de los hechos reales. Sin embargo, dada su orientación económica, autores como Mattessich (1964, citado por Mejía, 2005, 140) han considerado la contabilidad desde concepciones pragmáticas y modos de actuar cuyo único objetivo consiste en preparar estados financieros que sirvan de base para hacer proyecciones a futuro. No obstante, hay estudiosos de la teoría contable que defienden el enfoque crítico social de la disciplina, tales comoMachado (1991), Sarmiento (1996), Ariza (1996) y Franco (2003). Entre todos, se destacan Gómez y Ospina (2009), quienes plantean:

La Contabilidad no solamente actúa como medio, sino que se constituye en un conocimiento que amplifica, modifica, re-contextualiza y determina ideas, prácticas institucionales, estructuras simbólicas y morales, e intereses y visiones del mundo (pp.14$15)$.

De ello se establece que cambiar la visión reduccionista de la contabilidad implica una ruptura de pensamiento lineal que conlleva a una transformación en las metodologías para conducir los procesos de formación del contador. En esta vía han surgido autores como Túa (1997), García (2000, p. 2) y Cañibano 1997, p.4), que han abierto discusiones sobre la construcción de una teoría general de la contabilidad. . y todo esto exige la materialización de intenciones misionales de las universidades, a través de acciones que promuevan un sentido profundo de reflexión analítica y sintética que rompa, por fin, las estructuras de la enseñanza 
centrada en el "adiestramiento" (Loaiza, 2013).

Para lograr este fin, se requiere, en concreto, promover en el docente una visión inter y transdisciplinar del proceso de enseñanza de la teoría, así como un enfoque plurivalente. A pesar de ello, Gómez (2007, p. 5) plantea una realidad diferente cuando se refiere a la tendencia de los postgrados de la carrera ofertados, que apuntan a la profesionalización y espeficidad técnica de los oficios. En cuanto al estudiante, sus intereses se inclinan más a la práctica que al estudio analítico de la teoría. Se tiene la errada percepción de que saber de contabilidad es conocer la norma y saber aplicarla, dando pie a la utilización de la misma "por el camino cuantitativo donde lo importante son las cifras y su equilibrio sin tomar en cuenta el entorno donde se producen" (Stephens, citado por Casal y Viloria, 2002, p.30), pero con desconocimiento de las bases que le dan razón de ser a su actuar.

Autores como Popper (1982, p. 65), Franco (1995, p. 234); y Suárez, (2008, p. 113) coinciden en definir la teoría contable como un conjunto coherente de principios hipotéticos, conceptuales y pragmáticos en forma de redes, que permiten racionalizar y explicar el mundo definiendo marcos conceptuales para la práctica contable, cuya función es predecir acciones futuras. Sin lugar a dudas, esta interpretación ha sido producto de un proceso de investigación que busca pensar la contabilidad desde múltiples referentes, para hacerla más comprensible y elevar su estatus de simple técnica a disciplina y ciencia contable.

En Colombia, la teoría contable llegó con la institucionalización de la profesión del contador tras la 
promulgación de la ley 145 de 1960, siendo las universidades los espacios encargados de albergar y promover el debate para dinamizarla. Rueda (2007) y Valero \& Patiño (2012) afirman que desde entonces se observa escaso avance en los logros de la enseñanza de la teoría contable, evidenciándose débil formación de profesionales ante el reto de afrontar intervenciones sociales basadas en el conocimiento de la ciencia en contexto. Por ende, la teoría que fundamenta la disciplina contable no se desarrolla con la misma velocidad de la práctica. Los débiles procesos de investigación relacionados con estas temáticas giran en torno a la estructura conceptual de la teoría contable y a los usos instrumentales de la contabilidad en el entorno en el que actúa. Bauman (2011, p.14) precisa: "aunque se muestran importantes avances en estos temas, estos raramente acaban incidiendo sobre la línea central de las disciplinas académicas o sobre la vida cultural en general".

De acuerdo con Urrego (citado por Gómez, 2012): “La educación como función social atiende a la formación de sujetos que aporten al desarrollo social, por lo tanto, no sólo la educación universitaria tiene el compromiso de formar personas, sino que todo el sistema educativo debe aportar a la construcción de sujetos sociales" (p. 95). Pero este aspecto puede considerarse la mayor deficiencia de la contabilidad, área en la que se le llama teórico o investigador a cualquiera que publique libros sobre temas contables, aun cuando lo que comunica carezca de pertinencia (Rueda, 2007).

recientemente, se han conceptualizado los desarrollos teóricos de la contabilidad desconociéndose que estos son producto del "pensamiento intersubjetivo" con un 
sentido crítico social, el cual se ha propiciado por la interacción de los contables con otros actores sociales. De allí que todavía se minimice su importancia en la formación del contador público, lo que, al fin y al cabo, hace imperceptible su soporte básico en el ejercicio profesional y el deber de enfocar los problemas en forma analítica, lógica y coherente "para comprender la realidad social y organizacional reconfigurando categorías e instrumentos para intervenir en el mundo" (Gómez \& Ospina, 2009).

El panorama es mucho más sombrío si "se considera que algunas universidades aún no han incorporado la asignatura de Teoría Contable al programa de contaduría como proceso de reflexión en torno a la naturaleza de la contabilidad", plantean Gómez et al (2009, p. 21). En estas condiciones, es indudable la necesidad que tiene el estudiante de una formación continua para reunir conocimientos separados que posibiliten una forma más abierta de conocer y pensar complejamente, identificando el tejido común de sus relaciones (Morin, 2011, p. 148).

Lo anterior también trae consigo la necesidad de abordar la realidad a partir del estudio de la teoría, esto es, de revisar sus enfoques paradigmáticos, métodos, conceptos para obtener un conocimiento profundo del mismo; lo que Tua $(1995,37)$ señala como la necesidad de disponer de un mínimo conjunto de fundamentos de epistemología contable para razonar y aplicar la teoría, y, así, convertir al estudiante de las ciencias contables en un agente de cambio social (Convenio ICFES-FIDESC, 2000).

En el escenario actual, debido a la adopción de las llamadas Normas Internacionales de Información 
Financiera (NIIF), este enfoque se profundiza en la reflexión, toda vez que introduce características orientadas a la elaboración de informes financieros de manera que sirvan de guía para seleccionar métodos contables alternativos, de informar o revelar información útil para la toma de decisiones, las cuales requieren apoyarse en fundamentos epistemológicos que permitan concebir la función de la empresa como eje del desarrollo social. Con palabras de Ortiz (2010), que posibiliten "interpretar nuevas opciones de distribución de la riqueza para lograr una sociedad más justa y equilibrada" ( $\mathrm{p}$. 243).

Con la adopción de las NIIF, se intenta superar, por otra parte, el paradigma dominante, promoviendo de manera casi forzosa la utilización de las denominadas Notas a los Estados Financieros para revelar o ampliar el origen y el significado de los elementos presentes en los estados financieros, y así prever actuaciones futuras para conducir los resultados deseados. Precisamente, en el tema de la contabilidad internacional toma especial relevancia la teoría contable como medio de interpretación para comprender y contextualizar el lenguaje especializado que allí se maneja:

El lenguaje contable mundial no se "vulgariza", lo cual cuestiona fuertemente desde la Teoría Contable la pertinencia social del conocimiento científico que, por definición, busca constituirse en sentido común bajo esta Teoría Contable. El deber del científico crítico es "traducir" el mensaje técnico especializado y ponerlo al servicio de todos, "lo que se deja decir, se deja decir claramente [...] el desarrollo tecnológico debe traducirse en sabiduría de la vida. (Rueda, 2010, p. 213).

Del anterior planteamiento puede inferirse que la teoría aplicada a la contabilidad es una actividad lógica $\mathrm{y}$ racional, que apoya la explicación de los hechos económicos, mediante una serie 
de definiciones, principios, reglas, criterios y proposiciones, incluidos axiomas y teoremas, conformando su marco de referencia para discernir y comprender la naturaleza del saber contable con fundamento en las escuelas del pensamiento. Ello posibilita evaluar las prácticas contables desde una mirada crítica, reflexiva, de permanente duda, donde no se pretende tener todo claro. Así se garantiza el desarrollo de la práctica contable y la creación de nuevas teorías (Gómez et al, 2004).

A manera de ilustración de la aplicación de la teoría contable se presenta una caracterización de la partida doble apoyada en Matessich (1964, citado por Gómez et al, 2007), quien expresa: "la partida doble no solo se manifiesta en valores monetarios, existen otras expresiones de dualidad" (pp. 33-36), la mirada estrecha es el resultado de su visión centrada en aspectos normativos y comerciales con total abandono de los aspectos científicos de la enseñanza de la contabilidad. Los aportes de varios tratadistas permiten listar y sintetizar el potencial de la partida doble en seis grandes logros socio-contextuales y metodológicos señalados por Demski, Fellingham, Yujiljiri y Sunder (2002, p. 160) :

Primero: Separa la propiedad individual de los recursos destinados a la actividad comercial. Segundo: antecede $\mathrm{y}$ origina la necesidad jurídica de reconocer la existencia de entes ficticios o personas jurídicas. Tercero: sintetiza e instrumentaliza la dinámica propia del modo de producción capitalista (aumento del capital inicial por medio de la utilidad). Cuarto: sintetiza la relación de equilibrio entre Fuentes y Aplicaciones, Quinto: articula representaciones de flujos (o cuentas nominales) con representaciones de stocks (o cuentas reales). Sexto: articula representaciones de recursos físicos $y$ de recursos financieros, lo que se ha denominado como la capacidad de homogeneización de la contabilidad. 
De este modo, en la teoría contable, convergen dos aspectos intrínsecamente ligados, uno como consecuencia directa de la aplicación contextualizada de la partida doble en contabilidad, y otro que establece relaciones metodológicas y permite producir nuevas representaciones de la realidad.

En esta misma línea, como resultado de estudios previos, se habla de la necesidad de renovar los fundamentos teóricos de la partida doble por considerarse que presenta restricciones e inexactitudes y que la información reflejada es la de un estado pasado, de eventos ya ocurridos sin predicciones de su impacto. En respuesta a ello, autores como Ijiri (1989), presentan el método de partida triple, que no solo explica dónde está el ente y dónde ha ido, sino cuál es su curso. Para esto, se emplean datos históricos o predictivos, lo que implica una nueva estructura contable que, además de las columnas del débito y el crédito, comprende otra para la predicción o método trébito, de manera que se puedan conocer, medir y triangular las variables cuantitativas y cualitativas internas y externas que producen alteraciones en los estados financieros.

Como puede verse, son muchos los conocimientos contables que se ignoran de la creciente ciencia contable, y en ella la utilidad y la toma de decisiones se convierten en palabras claves. Por ejemplo, hasta hace unas décadas era imposible que los estados financieros incluyeran datos referidos al capital intelectual, hoy se aplica el modelo elaborado por Edvison y Malón (1999). También Kaplan y Norton (2000) han elaborado un modelo conocido como The Balanced Bussiness Scorecard, para definir indicadores financieros y no financieros, en tanto que se han desarrollado otros, como El monitor de 
Activos Intangibles de Karl E. Sveiby

(1997) y “El Modelo de Dirección

Estratégica por Competencias (Bueno, 1998).

\section{CONCLUSIONES}

\section{PERSPECTIVAS}

\section{Realidades en la enseñabilidad de la}

\section{teoría contable}

Rojas, Gracia y Ariza (2002), así

como Cardona \& Zapata (2005), entre otros, han planteado propuestas para mejorar las estructuras curriculares, al mismo tiempo que han evidenciado profundas debilidades en cuanto a la pedagogía y la didáctica en el proceso de enseñanza. $\mathrm{Y}$ es que la enseñanza de los fenómenos contables se ha orientado tradicionalmente a lo pragmático para dar respuesta al entorno de los negocios. En este marco, el enfoque de la complejidad aparece como medio para romper la reducción del pensamiento operativo, considerando su dimensión filosófica, social y política, en articulación con la ética como pensamiento axiológico necesario en todo proceso formativo. Sobre este tópico, Zaá (2010) puntualiza:

En esta nueva sociedad pugna por instaurarse una nueva razón con base en el nuevo paradigma de la ciencia, la complejidad dialógica, recursiva, auto ética y hologramática de la realidad, que nos ha permitido observar fenómenos y mensajes ocultos en las dimensiones de la realidad que hasta ahora no habían sido captadas con una visión cartesiana. $\quad$...la objetividad frente a la intersubjetividad, la estática frente al dinamismo permanente de los fenómenos, los valores discretos frente a los números borrosos, la cuantificación frente a la explicación, etc. (p. 21).

En función de esta premisa, y en su afán por descubrir un camino que conduzca desde las hipótesis de la ciencia contable hasta la definición de reglas concretas que orienten la elaboración de información, la teoría contable ha intentado la axiomatización a partir de Paton (1922), Palomba (1950-1952), 
Devine (1952) yLittleton (1958), pero tal propósito no se ha logrado debido a que las cambiantes dinámicas de las sociedades no han permitido la estandarización de lo contable. Al respecto, vale la pena recordar las palabras de Morin (2003) quien afirma: "Una teoría no es la solución, es la posibilidad de tratar un problema... el método y la teoría son inseparables" ( $\mathrm{p}$. 20).

Adecuando esto a nuestro problema, es claro que ninguna teoría puede abarcar y predecir todos los fenómenos contables, pero el hecho de que una sola teoría no pueda interpretar exactamente una realidad por sus múltiples variables, sugiere pensar en cómo desarrollar una nueva, acorde con las exigencias.

Hendriksen (1974) aduce:

La contabilidad no es positiva, ni normativa, ni es inductiva o deductiva, no tiene un método único, ni una manera exclusiva de abordar su estudio, asume una posición ecléctica en la cual toma de cada herramienta metodológica lo más útil, al igual que hacen otras ciencias humanas. Se descarta el monismo metodológico y se aboca por un pluralismo conceptual (p. 19).

Sin lugar a dudas, el nacimiento de nuevas teorías supone la necesidad de adaptar métodos y técnicas de aplicación cuyos contextos complejos para informar y tomar decisiones requiere formas cambiantes de pensamiento. No obstante, en la contabilidad se evidencia aún un débil nacimiento de teorías que dinamizan la profesión, pero desconocen la realidad cambiante del contexto en el cual tienen aplicación; en su carácter positivista, han instaurado como definitivas teorías fijadas como normas que orientan la práctica contable. Marx (citado por Morin) planteaba sobre este particular: "las doctrinas que se niegan a morir, se cierran a los argumentos contrarios y se refieren siempre al pensamiento infalible de su fundador" (p. 149). 
A tono con ello, Quintar (2007)

afirma:

El sentido en esta práctica de la enseñanza con sentido no es promover "pensar teórico", el pensar erudito, el tráfico de información, la verdad dada; por el contrario, aquí el sentido es reconstruir lazo en la relación sujeto / sujeto / realidad; es hacer del sentido de la recuperación de sentido un espacio de construcción de resistencia por la vida, es enseñar y aprender promoviendo $\mathrm{y}$ provocando el "saber situarse en la realidad como una tarea imperativa". En esta opción: realidad, sujeto, subjetividad, historicidad, potenciación y dialéctica como articulación se conforman en una matriz epistémica, en una estructura conceptual y/o categorial que se erige en dispositivos analíticos para leer la realidad y re significar teorías, haciendo un uso crítico de las mismas (p. 25).

Con esta nueva visión de la realidad se abandona el mundo de la certeza, la razón pura y práctica, entrando al mundo de la incertidumbre, del cual emerge el conocimiento trascendente que asume expresiones mucho más abiertas y llenas de sentido. Esto es, expresiones que se han construido desde lo sensible, apoyándose en métodos como la teoría crítica, el círculo hermenéutico, la teoría analítica, la teoría de la acción comunicativa, entre otros, que conciben la realidad como un todo, conectada por relaciones infinitas cuyo abordaje permite una mayor comprensión del objeto y cuyo discernimiento requiere un mejor adiestramiento del educando para detectarlas, razonarlas, analizarlas y relacionarlas. Al respecto, Torres \& Lam (2012) señalan:

En Latinoamérica algunas facultades de contabilidad no exigen a sus estudiantes hábitos de lectura. Sus egresados no demuestran pasión por la lectura. Desde estudiante el contador público competitivo tiene que ser constante lector de teorías, no sólo de contabilidad, sino de aquellas que tienen que ver con la organización $\mathrm{y}$ funcionamiento de la empresa y con los casos prácticos que tienen que resolverse en la organización (p. 45). 
De igual manera, se deben desarrollar las habilidades de escritura, para lo cual se destacan técnicas como la construcción de ensayos argumentativos y monografías, que Torres et al (2012) consideran como el medio que permite al estudiante convertirse en un "investigador potencial de la contabilidad" (p. 45).

Por otra parte, ante los desarrollos tecnológicos actuales, el profesional contable o "Contador" ha de convertirse en "Gestor de conocimiento e información contable", una labor de calidad que implica un conocimiento a fondo de la contabilidad para emplear sus conocimientos e interactuar inter $y$ trandisciplinariamente en el mundo globalizado (Lozano, 2011).

Es de esperar que ante ello la teoría crítica de la contabilidad tome especial relevancia, lo que conlleva nuevas formas de enfocar soluciones, en aras de desarrolla una contabilidad alternativa, no limitada a lo normativo, sino más bien a una apertura de pensamiento que promueva la integración de saberes para la solución de problemas complejos. Buena parte de lo expresado se centra en la pretendida objetividad y capacidad predictiva exigida a la contabilidad como condición para declarar su pertinencia social y legitimidad del conocimiento científico que promueve. Es así como una teoría contable con pensamiento crítico obra al servicio de una necesidad real para superarla, convirtiéndose en la alternativa con mayor probabilidad de éxito para la gestión gerencial.

En oposición a lo que se ha pensado de manera tradicional, los gerentes de hoy requieren, además del análisis, poseer habilidades para identificar problemas y oportunidades en 
ocasiones imperceptibles en el entorno de las organizaciones. "El éxito de los directivos dependerá en última instancia de su capacidad para prever los problemas antes de que ocurran..." (Stoner, 1989, citado por Codina, 2002, p. 23). Ello implica poseer conocimiento del medio organizacional y sus modelos de representación, así como habilidades para actuar con visión futurista "en el marco de la epistemología, la ontología, las matemáticas y la cibernética, entre otras" (Machado, 2009, p. 53).

\section{Infortunadamente, el proceso} formativo del contable ha enfatizado en la operación y descuidado la parte cualitativa para proyecciones y toma de decisiones, trascendiendo en resultados de investigación débiles, que no generan rupturas de pensamiento $y$, en consecuencia, tampoco elevan el nivel científico de la contabilidad. La madurez de esta ciencia se halla ligada al progreso de la teoría contable, que se ha enmarcado, sobre todo, en el enfoque cuantitativo de métodos estadísticos, que emplean la comprobación de hipótesis por comparación, y que requieren como complemento de articulación el enfoque cualitativo, sin el cual se producen descubrimientos sesgados por la parametrización.

En apoyo a lo expuesto, Machado (2012) afirma:

Los Contables (académicos, investigadores y practicantes) deben producir conocimiento socialmente útil más que datos o información procesada, sobre la realidad financiera de las organizaciones, estableciendo vínculos de la situación actual de estas con las posibilidades de su contexto local, regional, nacional y de las naciones latinoamericanas, en busca de generar condiciones de confianza y transparencia en su labor profesional así como las profundas transformaciones requeridas ante los asuntos críticos sociales (pobreza, corrupción, insolidaridad, etc.) que ponen en duda los tradicionales supuestos de orden $\mathrm{y}$ progreso, así como la legitimidad de los saberes que los solventan (p. 235). 
Lo anterior sugiere la siguiente pregunta: ¿cuál es el conocimiento pertinente en la formación del contador público? Pregunta ante la cual surge respuestas como la de Morin (2011, p. 151): "un conocimiento no es tanto más pertinente cuanto más informaciones contiene o cuanto más rigurosamente organizado está en forma matemática; es pertinente si sabe situarse en su contexto y, más allá, en el conjunto con el cual está relacionado".. Por consiguiente, el proceso de enseñanza-aprendizaje de la teoría contable requiere una pedagogía especial, que incluya diferentes caminos de abordaje a la contabilidad desde lo formativo, y que así logre en el estudiante un verdadero aprendizaje significativo y aporte a la construcción de la disciplina.

$$
\text { Recientemente, ha surgido la }
$$
llamada "Contalogía", como declaración del carácter científico de la contabilidad y base del racionalismo aplicado. Esta se cataloga a sí misma como la filosofía que sustenta la contabilidad, dándole soporte epistemológico y constituyéndose en el medio fundamental para determinar su naturaleza científica y garantizar tomas de decisiones fundamentadas. En correspondencia con ello, la formación de un profesional contable integral competente se logra mediante el desarrollo de conocimientos y habilidades requeridas por el contexto, aunado a características personales de cada individuo imposibles de enseñar (Stoner, 1989, citado por Codina, 2002).

Como se aprecia, esta nueva visión paradigmática exige nuevas formas de observación fenoménica, la cual se construye en un proceso de formación que propicie la comprensión e interpretación, el análisis, deconstrucción y reconstrucción de los fenómenos estudiados. Todo esto, a partir de un 
reconocimiento de la situación actual de nuestra disciplina, razonando más que desde la técnica misma, desde el fundamento, el origen, la evolución y sus consecuencias, pero también potenciando

\section{REFERENCIAS}

Ariza D. (2002). La Formación del contador público: Alternativa para interpretar y transformar un mundo complejo. Popayán: Universidad del Cauca. los planteamientos interdisciplinarios $\mathrm{y}$, por último, pero no menos importante, sensibilizando a nuestros docentes y estudiantes en el importante valor de la investigación (Tua, 1997, p. 901).

Bloom B. (1956). La taxonomía de Bloom y sus actualizaciones. Modificado el 01 de febrero de 2010, del sitio Web de Eduteka: Recuperado en: http://www.eduteka.org/Taxonomi aBloomCuadro.php3.

Bueno E. (1998). El capital Intangible como clave estratégica de la competencia Actual. Boletín de Estudios económicos, Vol. LIII, No 164. Pág. 221.

Cañibano L. (1997). La teoría actual de la Bauman, Z. (2011). Modernidad y contabilidad. Madrid. Ediciones holocausto. ( $6^{\mathrm{o}}$ Ed.). Madrid: Ice.

Sequitur. 
Convenio ICFES-FIDESC (2000). La Contaduría Pública del Nuevo Milenio.

Renovación Curricular, Bogotá: Ecue Ediciones.

Cardona J. Zapata M. (2005): Educación Contable Antecedente, actualidad y prospectiva. Universidad de Antioquia, Medellín.

Casal, R. y Viloria, N. (2002). La corriente positivista y su influencia en la ciencia contable. (Mérida). Actualidad Contable FACES, 05, 05, 07-22

Codina A. (2002). ¿Qué hacen los directivos y qué habilidades necesitan para un trabajo efectivo? Resumen y Análisis de Investigaciones. Centro de Estudios de Técnicas de
Dirección) de la Facultad de Contabilidad y Finanzas de la Universidad de La Habana
Cuervalo, H. (2009). Hechos y eventos de la responsabilidad social: memoria histórica desde la

Contabilidad: cuadernos de contabilidad 9 (25) 487-501.

Demski J., Yuji I, \& Sunder S. (2002). Accounting as a Microcosm. En: Some Toughts on the Intellectual Foundations of Accounting. 2001.

Devine T. (1952): Integration of Accounting and Economics in the Elementary Accounting Course. The Accounting Review, July, pp. 329-33 
Edvinsson, L; Malone, M.S. (1999). El capital intelectual. Gestión 2000. Barcelona.

Franco R. (2003). Hacia la contabilidad integral En: revista internacional Legis de Contabilidad y Auditoría, No. 16, julio- septiembre

Franco R. (1995). Reflexiones Contables. Tipografía y litografía Atenas Ltda.

Gracia L. (2002). Estado actual de la educación contable en Colombia. Universidad del Cauca. V.01, p. 85-111, 01, 2002 En: CCINCO.

Comp. (Varios autores). Popayán: Universidad del Cauca, pp. 85-111.
Gómez, Y. (2012). Educabilidad: Unare significación conceptual en contabilidad desde

La Educación crítica. Contaduría Universidad de Antioquia, 61, 91-110

Gómez, Y. (2012). Enseñabilidad y educabilidad de la contabilidad: primer acercamiento. XIII Asamblea ALAFEC 2012, Universidad de Buenos Aires Argentina. http://www.alafec unam.mx/memoria_xiii.php.

Gómez, M. \& Ospina, C. (2009) Ampliando las fronteras de la Disciplina Contable: una introducción para la Comprensión de los Ejemplares Heterodoxos. En: Avances Interdisciplinarios para una Compresión Crítica de la Contabilidad: Textos 
Paradigmáticos de las corrientes

heterodoxas. Medellín 2009. 13-

35.

Gómez M. (2007). a Comentarios sobre el aprendizaje construcción de la Teoría Contable. En Colombia Hendriksen S. (1974) Teoría de la Lúmina: Universidad de Manizalesv.7 fasc. p.129 153

Gómez M. (2007). Pensamiento único y contabilidad: la posibilidad de la Contabilidad Como disciplina moral. Revista Ciencias Económicas: Investigación y Reflexión, vol. XV, 2, diciembre, 2007, pp. 29-43, Universidad Militar Nueva Granada Colombia.

Gómez, M. (2004). Breve introducción al estado del arte de la orientación critica en la disciplina contable. Contaduría Universidad de Antioquia, 45, 115-132.
Gracia E. (2002). Estado Actual de la Educación Contable en Colombia. En: Del Hacer al Saber. Universidad del Cauca. Popayán. de Contabilidad.

México:

Ijiri Y. (1989). Momentum de contabilidad y teneduría de libros triple entrada: la exploración de la dinámica estructura de las mediciones contables, Estudios de Investigación Contable, vol. 31, American Accounting Association, Sarasot Kapklan R. y Norton D.(2000) “Cuadro de mando integral”. Gestión 2000. Barcelona. Pág. 105 y sgtes U.T.E.H.A. 
Lara J. (2002). Verdad, Responsabilidad social y Ética en Contabilidad. Ética y desafíos de la Contaduría Profesional. Ciencias Contables. Pontificia Universidad Javeriana.

Littleton A. (1958): "Accounting Rediscovered". The Accounting Review, pp.246-253.

Loaiza F. (2013). Corrientes educativas internacionales presentes en programas de contaduría pública. Cuadernos de contabilidad, $13 \quad$ (34) 189-215. Recuperado de: http://www.javeriana.edu.co/fcea/cuadern os_contab/vol14_n_34/vol14_34_7.pd

Lozano C. (2011), Revolución o evolución del árbol contable, XXII Congreso Nacional de contadores públicos del Perú

Machado, M. (2012). Del positivismo a la diversidad epistemológica: un paso necesario para la construcción de pensamiento contable. Contaduría Universidad de Antioquia (60,219).

Machado M. (2009). Contabilidad y realidad: Una relación crítica bajo el enfoque de la representación Actualidad Contable FACES Año $12 \mathrm{~N}^{\mathrm{o}}$ 19, Mérida. Venezuela (38-55).

Machado, A. (1991). La contabilidad una ciencia social, en Revista Contaduría U. de Antioquia, Nos 17 - 18, Medellín, Antioquia

Martínez, G. (2002). El rediseño curricular contable: entre lo profesional y lo disciplinal. Del hacer al saber. Editorial Universidad del Cauca 
Mejía, E. (2005). Introducción al pensamiento contable de Richard Mattessich. En: Revista Internacional de contabilidad \& auditoría. $N^{\circ} 24$.
Patton W. (1922): Accounting Theory, New York: Ronald Press.

Popper, K (1982) La lógica de la investigación científica. Madrid: Tecnos.

Morín E. (2011). La vía. México. Paidós.

Quintar E. (2007). Universidad, producción de

Morín y otros (2003), Educar en la era Planetaria, Capítulo I: El Método, Editorial Gedisa. F.Girard, \& N. Vallejo, Trads.) París: UNESCO

Ortiz B. (2010). La complejidad y la teoría contable. Criterio Libre, 8231. para la educación del futuro. (M. Vallejo,

conocimiento y formación en América Latina, Polis [En línea], $18 \mid 2007$, DOI: $10.4000 /$ polis.409

Morín, E. (1999). Los Siete Saberes necesarios Rojas, W. (2002). La Educación Contable al Servicio de la Afraternidad Económica Moderna. En: Del Hacer al Saber. Universidad del Cauca. Popayán.

Rojas W. (1996). "Consideraciones sobre

Palomba G. (1950): Introduzioneall'Economica. Napoli: Del Gaudio.- (1952): CicliStorici CicliEconomici. Napoli: Giannini. los alcances de la enseñanza de la teoría contable". En: Colombia, Cuadernos De Administración Ed: Facultad De Ciencias De 
La Administración Universidad

Del Valle v.23 fasc. N/A p.173 - 181.

Rueda, G. (2010). Contabilidad para la dignidad social. Los aportes de la teoría crítica para la construcción de una nueva dimensión internacional para la Contabilidad. Contaduría, Universidad de Antioquia, 57, 203-219.

Rueda, G. (2007). La investigación contable: vínculos ontológicos y las posibilidades de la investigación interpretativa. Contaduría Universidad de Antioquia, 50, 119-132.

Sarmiento, H. (1996). La urdimbre del Quipus, elementos para un abordaje crítico al objeto de estudio de la contabilidad, en
Revista Lúmina, de la facultad de Contaduría de la Universidad de Manizales, $\mathrm{N}^{\mathrm{o}} 01$ de diciembre 1996.

Suárez, J. (2008). Momentos estelares de la contabilidad. Bogotá. Ediciones Gran Colombianas.

Torres C. \& Lam W. (2012). Fundamentos epistemológicos de la contabilidad y su incidencia en la formación competitiva Del contador público. Universidad Externado de Colombia, Recuperado en: http://revistas.uexternado.edu.co/i ndex.php?journal=sotavento\&pag $\mathrm{e}=$

article \&op=view \&path $\% 5 B \% 5 D=3339 \&$ path $\% 5 B \% 5 \mathrm{D}=2989$ 
Túa J. (1997). Lecturas de teoría e $\underline{\text { http://investigacion }}$ investigación contable. Editorial erg.edu.ve/nex.

CIJF Centro Interamericano

Jurídico-Financiero. Medellín.

Tua J. (1995). Lecturas de teoría e investigación contable. Centro Interamericano Jurídico

Financiero. Medellín.

Colombia.1995. Página 219.

Valero G. \& Patiño R. (2012): Los grupos de investigación contable reconocidos por Colciencias, Cuad.

Contab. vol.13 no.32 Bogotá Jan./ June 2012.

Zaá, JR. (2010). Del discurso científico de la modernidad a una nueva normatividad en la construcción del conocimiento contable. Disponible en: 